medicine-man dispensed their arts. He tries to show us how in fundamental ways medical practice has remained unchanged for millennia, that the basic needs of patients and the old ways of practising medicine emerge as a natural consequence of social interaction. He argues that unless patients rise up and fight for their rights, doctors, who always have had the upper hand, will continue to exercise unreasonable control.

His argument from history is, however, sketchy and flawed. Anthropologists tell us that healers in tribal societies did quite different things in different cultures for their sick and dying. Levi-Strauss reports that the "shaman" in one society may act the self-conscious charlatan, reaping the rewards of power and prestige; in another, the healer may be someone who merely happens to know tribal lore. Moving on, Preston also forgets to inform us that mediaeval barbers and surgeons practised their trades in the same guild. Nor does he tell us that, until modern times, surgeons and chefs were in the same fraternity in the British navy. What bound the cooks, barbers and surgeons was not their natural social standing, but their knives and scissors - or perhaps the fact that food, hair and disease have always been and will always be with us.

By making us believe that the doctorpatient relationship is a natural feature of the human landscape, Preston stumbles and falls into the same myth-making apparatus that holds us all in its thrall. While he makes some recommendations for reform, he fails to see how he has adopted the same myths that have helped American doctors secure their power and privilege.

Preston would have been a better guide had he not turned the pages of his history book so far back. In the nineteenth century, American physicians practised alongside homeopaths, midwives and assorted fakers and purveyors of patent medicine. It was not until the early days of this century that doctors, under the political leadership of the American Medical Association, codified their position and under the slogans of standardization and faith in science, systematically excluded all but those who emerged from approved medical schools and who gained access to practice by passing state licensing exams (also controlled by the AMA and its local societies). By the 1920s, the AMA had established a complete monopoly over the practice of medicine in the United States.

In The Clay Pedestal, Thomas Preston has tackled a subject which demands close and critical scrutiny. But an analysis of medicine that merely reflects what patients already feel about their doctors, and neglects to tell them how doctors gained their power, says a lot about what we already know and nothing about what we do not.

Robert Ubell is the American Publisher of Nature.

\title{
Around the world of mental illness
}

\section{G. Morris Carstairs}

Psychiatry Around The Globe: $A$ Transcultural View. By Julian Leff. Pp.204. ISBN 0-8247-1532-2. (Dekker: 1981.) SwFr. 58.

THIS conspectus of mental illness "around the globe" has many of the attributes of those luxury cruises in which travellers combine the pleasures of exotic sightseeing with those of being instructed by experts about what they have seen. Dr Leff is an admirable tour guide. He handles statistics with respect but is always ready to point out their limitations as well as their contributions to knowledge. As befits an epidemiologist, he first scrutinizes the methodology of any survey and only then discusses its findings.

His global tour de l'horizon is clearly presented, under four major headings: (i) Do psychiatric conditions look the same in different countries? (ii) Do psychiatric conditions have the same frequency in different countries? (iii) Are psychiatric conditions treated differently in different countries? (iv) Do psychiatric conditions have a different course in different countries?

These four parts are followed by a final section discussing the mental health of immigrants in general, and of West Indian and Asian immigrants in Britain in particular. He gives us interesting summaries of research findings in each of these areas of inquiry. From start to finish one basic question keeps recurring, namely: if multiple culture-specific factors enter into the perception, treatment and outcome of psychiatric illnesses, then how meaningful is it to make cross-cultural comparisons of these illnesses?

There is now ample evidence that both organic and functional psychoses can be identified in every society; but even in respect of these major illnesses we have to proceed cautiously in interpreting symptoms in the same way as we would do in the West. As Leff puts it: "It is clear that delusions and hallucinations, the main symptoms of psychotic illness, can only be judged as present in relation to the patient's cultural milieu',. This still leaves us uncertain as to just how such symptoms are to be evaluated.

A large part of this book is thus devoted to demonstrating ways in which the procedures of psychiatric diagnosis are being made more valid and reliable than they have been in the past. An interesting example is the US/UK Project which had its origin in an observation of the biostatistician, Morton Kramer. $\mathrm{He}$ pointed out that, if their respective data were to be believed, then the first admission rates for manic depressive psychoses in the age group 55 to 64 were twenty times higher in Britain than in the USA. Could this really be true?
In order to put the question to the test it was necessary to construct an objective, systematic interview schedule and to train a team of six psychiatrists (four British and two American) in its use. The instrument chosen was Professor John Wing's Present State Examination (PSE). The team used it to ascertain the "project diagnosis" for consecutive series of patients admitted to hospitals serving London and New York. When they did so, the previously wide discrepancies in diagnoses became very much reduced. Analysis of the results showed that hospital doctors in New York were accustomed to use a much wider concept of schizophrenia than that used in London, as a result of which they included under the label of schizophrenia many patients who would be diagnosed as manicdepressive in London.

This was the first international use of the PSE. Its next major deployment was in the WHO-sponsored International Pilot Study of Schizophrenia (IPSS) in which teams in nine countries (the USA, Britain, Colombia, Czechoslovakia, Denmark, India, Nigeria, Russia and Taiwan) collaborated in collecting cohorts of patients who met certain defined criteria, assessing them in terms of the PSE and then following them up for a period of five years. Here the PSE could be used in its original form only in Britain and the USA; in each of the seven other countries it had to be translated into the local language.

This international study (which still continues) has produced some interesting findings; among them, the demonstration that patients with specified "core symptoms" of schizophrenia could be found in every country; and also that the final diagnostic judgements (whose criteria were not laid down) were fairly uniform in seven countries but widely divergent in the other two, namely the USA and Russia. In the former, a cultural emphasis on Freudian concepts led to a wide concept of schizophrenia so that many cases were included under this heading which would not be so regarded in the other countries. In Russia, diagnostic practice was - and still is - dominated by Professor Snezhnevsky's theory that schizophrenia presents in three forms, much attention being paid to the postulated course of the disease. Many patients were diagnosed as schizophrenic because although they did not yet show major symptoms it was predicted that they would eventually do so.

Dr Leff is candid about the large obstacles which hinder cross-cultural agreement about certain of the symptoms of mental illness, particularly in the neuroses. He devotes a chapter to discussing "The language of emotion", pointing out that there are some languages in which there are no single terms for “depression" or "anxiety". In translating 
an instrument such as the PSE into Yoruba, for example, recourse has to be made to metaphors in common use such as "the heart is weak", for depression, and "the heart is not at rest", for anxiety. One cannot be sure that these phrases correspond at all precisely with the English words.

Not only are some languages richer than others in the language of emotion: even within cultures members of underprivileged groups may have less welldeveloped abilities to recognize or to express nuances of feeling (as Basil Bernstein has demonstrated in British society).

Dr Leff is dedicated to the use of standardized instruments, accompanied by glossaries and sets of instructions as the best way to enable different observers to recognize and record symptoms in the same way. Usually he remains keenly aware of the difficulties which still remain, but very occasionally he lapses as when, after completing a review of a number of surveys of psychiatric illness in Asian countries he draws attention to the wide differences in the prevalence rates for neurosis, and comments: "We can infer that the very highest rates found in the Asian surveys are likely to represent the most realistic estimates of the true prevalence of the neuroses".

Normally, he would be the first to question the concept of the "true prevalence" of neuroses. He knows that minor neurotic symptoms are very common in normal subjects. Determining at what levels of frequency and severity these symptoms should constitute an illness is a judgemental decision. Until some less subjective indicator can be found, the prevalence of neuroses can only be measured in terms of stated, hopefully relevant, operational criteria. As yet there is no such thing as the "true prevalence" of neuroses and perhaps never will be.

The chapters on the mental health of immigrants to the USA and to Britain are interesting not only for their findings (that, in general, immigrants show a high rate of incidence of schizophrenia but that Asian immigrants attend their general practitioners for minor complaints less often than do their British neighbours), but also because they show that useful crosscultural research can now be carried out within the British Isles and USA. One factor, however, appears to have been overlooked. This is Srole et al.'s report, Mental Health in the Metropolis: The Midtown Manhattan Study (McGraw-Hill, 1962), which found very high rates of psychiatric disorders among immigrants but only among poor immigrants. The much smaller number of rich immigrants did not experience an excess of these disorders.

The final chapter tells us, in grim detail, about the many handicaps which an Asian immigrant physician has to contend with in trying to advance his career in a Western country: these are economic, linguistic, social, educational as well as professional. Having taken a special interest in the welfare of overseas postgraduate students at the Institute of Psychiatry, London, Dr Leff knows what he is talking about; but here he seems to present a totally gloomy picture. Every experience of the immigrant doctor is a negative one, culminating for some in a neurotic or psychotic breakdown, with all their disastrous implications. Is there no gleam of light at all in the experience of our immigrant trainees? Surely we can all remember some exceptions, men and women who have earned the friendship and respect of their British or North American colleagues and have embarked upon an academic or clinical career. Admittedly, they are in the minority. This chapter, although tangential to the rest of the book, will serve a useful purpose by reminding us about the difficult lot of our overseas trainees.

G. Morris Carstairs is currently a Fellow of the Woodrow Wilson Center, Washington DC, and recently spent three years in India as a visiting professor of psychiatry.

\section{Art of insects}

\section{J.L. Cloudsley-Thompson}

Insects Etc.: An Anthology of Arthropods Featuring A Bounty of Beetles. Pp.108. ISBN 0-933920-25-3. (Hudson Hills: 1981.) $\$ 50$.

WHAT is there in common between Edgar Allan Poe, Jonathan Swift and Raoul Hausmann? The answer, immediately revealed by perusal of the literary anthology with which P.A. Gette enlivens this unusual book, is that they were not good observers of insects. Like Salvador Dali's pre-1950 ants, Hausmann's houseflies had only four legs, Swift's were endowed with stings and Poe confused bugs with beetles. Far more observant were Lewis Carroll and Vladimir Nabokov and, of course, Réaumur, Thoreau and JeanHenri Fabre. Buffon was evidently a careless writer: "The insect is a small animal without blood. The latter [sic] can be divided into large and small kinds".

Literary activities, like art, are not necessarily marred by inaccuracy such as this. Less excusable, however, are the errors of nomenclature and spelling apparent in the names of the various arthropods portrayed. For instance, Argiope bruennichi (mis-spelled) is an impressive, striped, orb-web spinner, but it is not the zebra spider. This name has always been reserved for Salticus scenicus the little jumping spider, common on walls and fences throughout Europe, Asia and America. Again, the scorpion is not Buthus occitanus as stated but, unmistakably, Scorpio maurus, the classical "scorpio" of antiquity.

The 34 dazzling, large-format colour plates are taken from photo-realistic paintings by Bernard Durin, without doubt an unusually gifted illustrator in the great tradition of Audubon, Edward Lear and Louis Agassiz Fluertes. Of 33 species illustrated (there is a close-up of the head of Zonocerus variegatus in addition to a painting of the whole grasshopper), 16 are beetles, 5 grasshoppers, 6 Hemiptera, 3 Hymenoptera, a single butterfly (the peacock), a spider and a scorpion. The species depicted are, as might be expected, large, brightly coloured and sometimes bizarre. The paintings illustrating them are breathtaking. Durin is not only a meticulous observer but he really succeeds in creating the illusion of irridescence in his beetles and in making insects' wings look transparent. A commentary on each of the arthropods illustrated, with information about various aspects of its behaviour, life cycle, food, enemies and folklore, is provided by Gerhard Scherer.

Zoologists are often so familiar with the subjects of their research that they overlook obvious questions posed by them. What are the functions of the extraordinary colours of some insects, why do others have such long legs or antennae, what are the advantages and drawbacks of their shapes and sizes? It might be worthwhile taking a second, thoughtful look at the subjects of Durin's paintings.

J.L. Cloudsley-Thompson is Professor of Zoology at Birkbeck College, University of London.

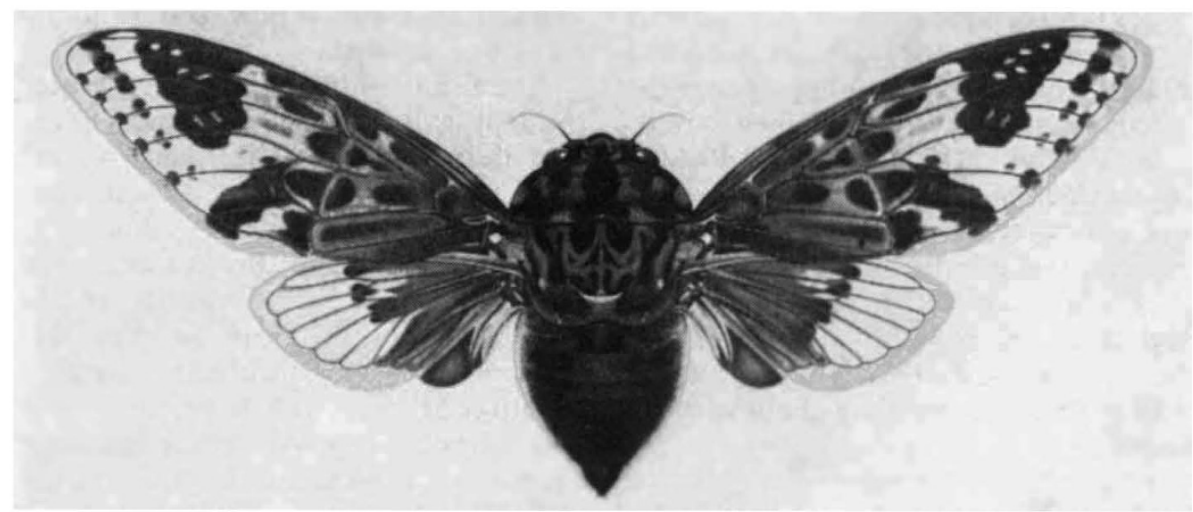

Pycna antinorii, the African cicada, painted by Bernard Durin. Reproduction in black and white.and reduction in size do scant justice to the picture. 